Corrigendum

\title{
Corrigendum to: Dual mechanisms for the regulation of brain- derived neurotrophic factor by valproic acid in neural progenitor cells
}

Hyun Myung Ko ${ }^{1, *}$, Yeonsun Jin ${ }^{2, *}$, Hyun Ho Park ${ }^{3}$, Jong Hyuk Lee ${ }^{4}$, Seung Hyo Jung ${ }^{5}$, So Young Choi ${ }^{6}$, Sung Hoon Lee $^{2}$, and Chan Young Shin ${ }^{7}$

${ }^{1}$ Department of Life Science, College of Science and Technology, Woosuk University, Jincheon 27841, ${ }^{2}$ Department of Pharmacology, College of Pharmacy, Chung-Ang University, Seoul 06974, ${ }^{3}$ School of Pharmacy, Chung-Ang University, Seoul 06974, ${ }^{4}$ Department of Pharmaceutical Engineering, College of Life and Health Science, Hoseo University, Asan 31499, ${ }^{5}$ Departments of Medicine, Research Institute of Medical Science, Konkuk University School of Medicine, Chungju 27478, ${ }^{6}$ Department of Biomedical Science \& Technology, Konkuk University, Seoul 05029, ${ }^{7}$ Departments of Pharmacology and Advanced Translational Medicine, School of Medicine, Konkuk University, Seoul 05029, Korea

*equal contribution

\section{Corrigendum to:}

Korean J Physiol Pharmacol. 2018;22(6):679-688.

http://doi.org/10.4196/kjpp.2018.22.6.679

The authors note that on page 685, the acknowledgement of "This study was supported by the National Research Foundation of Korea (NRF) grant funded by the Korea government (MEST) (No. 2017R1D1A1B03031920)," should instead appear as “This study was supported by the National Research Foundation of Korea (NRF) grant funded by the Korea government (MEST) (No. 2017R1D1A1B03031920) and Chung-Ang University Research Grants in 2017.” 